\title{
Erratum: New evaluation of the antiproton production cross section for cosmic ray studies [Phys. Rev. D 90, 085017 (2014)]
}

Mattia di Mauro, Fiorenza Donato, Andreas Goudelis, and Pasquale Dario Serpico

(Q) (Received 26 July 2018; published 10 August 2018)

DOI: 10.1103/PhysRevD.98.049901

In this paper, Eq. (10) reports the first line of Eq. (5) in Ref. [1]. However, the equation has been reported with inexact energy units. The correct expression, which matches the one in [1], is

$$
\sigma_{\text {in }}(s)=\sigma_{0}\left[1-0.62 e^{-\frac{E_{\text {inc }}(s)}{200}} \sin \left(\frac{10.9}{E_{\text {inc }}^{0.28}(s)}\right)\right]
$$

where the kinetic energy $E$ is in MeV. Also, an extra 0 had been mistyped in Table II as the first decimal digit in entry $D_{4}$. The entry should read 0.510 instead of 0.0501 . The corrected table follows:

\begin{tabular}{lcccccc}
\hline$D_{1}$ (error) & $D_{2}$ (error) & $D_{3}$ (error) & $D_{4}$ (error) & $D_{5}$ (error) & $D_{6}$ (error) & $D_{7}$ (error) \\
\hline $4.22(0.66)$ & $3.435(0.016)$ & $0.0067(0.0014)$ & $0.510(0.050)$ & $3.609(0.021)$ & $0.0209(0.0010)$ & $3.086(0.083)$ \\
\hline
\end{tabular}

None of the typos affect the results reported in this paper. We thank R. H. Bernstein for spotting the two typos.

[1] R. P. Duperray, C.-Y. Huang, K. V. Protasov, and M. Buenerd, Parameterization of the antiproton inclusive production cross-section on nuclei, Phys. Rev. D 68, 094017 (2003). 